\title{
My approach to intraductal lesions of the prostate gland
}

\author{
M Pickup, T H Van der Kwast
}

J Clin Pathol 2007;60:856-865. doi: 10.1136/icp.2006.043224

The morphologically heterogeneous (intra)ductal lesions of the prostate frequently present a diagnostic challenge, particularly when found within prostate needle biopsies. By current convention, all high-grade intra-acinar and intraductal neoplastic lesions of prostatic origin fall under the diagnostic umbrella term: prostatic intraepithelial neoplasm (PIN). Although a long-standing contentious issue, some lesions currently adhering to the diagnostic criteria of PIN may actually represent the intraductal spread of (generally high grade) invasive cancer. Illustrating this fact, the well-described ductal subtype of prostatic adenocarcinoma is frequently associated with conventional-type acinar adenocarcinoma, and has a tendency to propagate within adjacent intact prostatic ducts. Clearly, the misdiagnosis of lesions representing invasive disease as preinvasive has the potential for unfavourable clinical sequelae. As yet, however, many of these lesions have escaped the establishment of reliable morphologic criteria or immunohistochemical differentiation for diagnosis. By defining stringent architectural and cytonuclear features specific for each of these lesions, it may be feasible to separate potentially sinister lesions from the subset of traditional (preinvasive) PIN lesions with limited clinical urgency. This review discusses the (intra)ductal lesions of the prostate, along with their differential diagnoses. Given the current state of knowledge, a pragmatic approach to their effective reporting is outlined, taking into consideration the clinical implications, as well as current guidelines for treatment and follow-up.

See end of article for authors' affiliations

Correspondence to: Professor TH van der Kwast Department of Pathology,

11 th Floor, University Health Network, 200

Elizabeth Street, Toronto, Ontario, Canada M5G 2C4; theo.vdkwasł@uhn. on.ca

Accepted

20 December 2006

Published Online First

19 January 2007
$\mathrm{T}$ he ductal lesions of the prostate and their mimics span a spectrum from benign to premalignant through to, frankly, invasive disease. Adding to the complexity is the lack of distinct histological differences between prostatic ducts and acini. Indeed, even in benign tissue, transverse sections of prostatic ducts are indistinguishable from acini. Lesions with ductal morphology include ductal adenocarcinoma, prostatic intraepithelial neoplasia, as well as the intraductal spread of an adjacent, generally highgrade (Gleason score 8-10) adenocarcinoma. The histological features, immunohistochemical profiles and the nomenclature of these lesions are a topic of constant debate, and a considerable amount of disagreement exists within the sphere of genitourinary pathology. This article reviews the prostatic lesions with ductal morphology, as well as their mimics, in the hope of guiding anatomical pathologists towards accurate diagnosis and effective reporting of these frequently challenging lesions.

To understand the pathology of lesions with ductal morphology, one must first define the term "ductal morphology". One approach takes into consideration the microscopic anatomy of prostatic ducts. Each of the three anatomically distinct zones of the prostate has its own set of periurethral main prostatic ducts lined by several layers of urothelial-like cells, reminiscent of the urothelium. ${ }^{1}$ The peri-urethral ducts give off branches, with tributaries adopting the epithelial morphology of prostatic acini as they progress upstream from the urethra (fig 1). The tall columnar cells lining the larger ducts resemble the cells lining the smaller ducts and acini in their staining patterns with prostate-specific antigen (PSA) and prostatic acid phosphatase (PAP). ${ }^{2}$ Thus, following this approach, prostatic ductal lesions are derived from the large periurethral ducts, and the lesional cells tend to share their tall columnar morphology. An alternative approach makes a morphological analogy between prostatic ductal lesions and the in situ neoplasias of the breast. Using the breast analogy as a framework, the ductal lesions of the prostate can be identified by their well-circumscribed rounded contours (occasionally with identifiable branching) and their larger calibre than the surrounding acinar structures. The range of neoplastic lesions of the prostate with ductal morphology and their differential diagnoses are listed in table 1 , which serves as the basis for this review.

\section{PROSTATIC INTRAEPITHELIAL NEOPLASIA Definition and morphology}

High-grade prostatic intraepithelial neoplasia (PIN) is the term used to denote the presence of dysplastic features in the luminal cells lining prostatic glands or ducts. The hallmark of PIN is the presence of enlarged nucleoli in cells lining prostatic glands or ducts with a luminal (but not basal) cell morphology and location. ${ }^{3-5}$ At low power, attention is drawn to glands and ducts involved by PIN as a result of their slightly more basophilic appearance when compared with unaffected structures. The basophilia is due to both nuclear crowding and increased cytoplasmic density. The arrangement of acini, however, retains a benign pattern. PIN may assume variable architectural patterns, and, frequently, different patterns are found within the same specimen. Several architectural variants of PIN have been described (fig 2). Although the tufted and micropapillary

Abbreviations: AMACR, $\alpha$-methylacyl coenzyme A racemase; PAP, prostatic acid phosphatase; PIN, prostatic intraepithelial neoplasm; PSA, prostate-specific antigen 

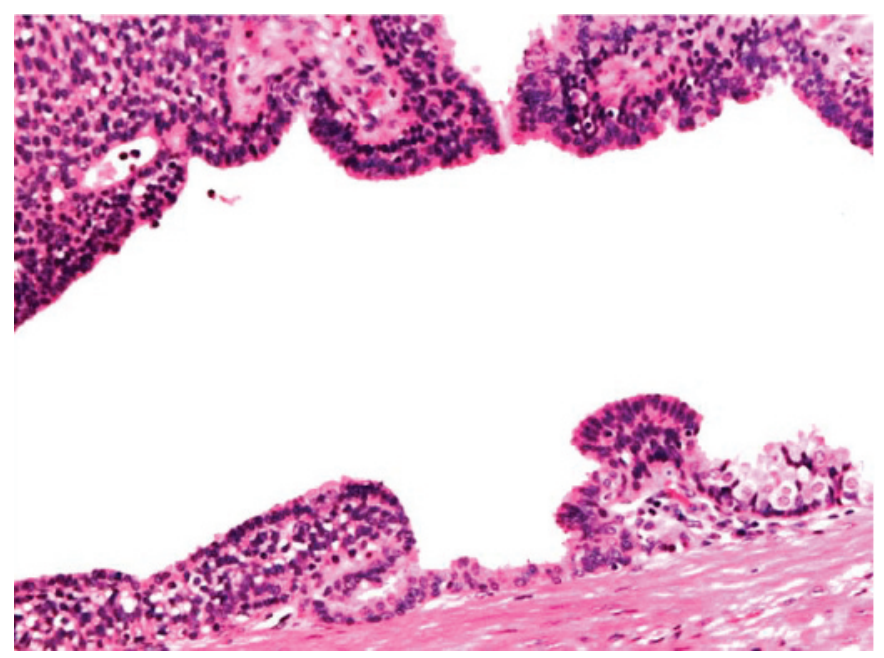

Figure 1 Longitudinal section of the larger main prostatic duct, lined by mutiple layers of epithelial cells and an inner layer of columnar cells. Outpouchings are lined by prostatic secretory cells.

patterns are most common, ${ }^{6}$ other patterns show stratification of dysplastic cells, sometimes filling a large part of the glandular or ductal lumen. The latter variants include cribriform, solid and comedo-type PIN. ${ }^{7-9}$ The analogy (at least in terms of morphology) with the four classical variants of ductal carcinoma in situ of the breast (ie, micropapillary, cribriform, solid and comedocarcinoma) is obvious. Other, exceedingly uncommon variants of PIN (eg, small cell, signet cell, hobnail or inverted pattern, foamy cell $)^{10}{ }^{11}$ are beyond the scope of this review.

By consensus, all architectural variants of PIN are currently termed "PIN", and are so reported..$^{512}$ PIN is generally considered to represent the most common precursor lesion of prostatic adenocarcinoma. ${ }^{5}{ }^{14}$ In addition to sharing the predominantly peripheral distribution of adenocarcinoma, PIN is found in association with invasive carcinoma in $>70 \%$ of prostatectomy specimens. ${ }^{5}{ }^{15}$ In a study examining cystoprostatectomy specimens from men with bladder cancer, Montironi et $a l^{16}$ found that PIN was associated with prostate cancer in about $80 \%$ of cases, whereas specimens without concomitant prostate cancer had a significantly lower incidence of PIN, approaching 30\%. Furthermore, molecular-genetic changes and complementary DNA expression profiles found in PIN have been shown to closely mirror those of prostate cancers found within the same specimen. ${ }^{17-19}$ It should be noted that none of these molecular-genetic studies distinguished the different architectural variants of PIN, and they therefore do not provide specific information on the subset of lumen-spanning PIN.

Less certainty exists with respect to a precursor role of PIN in the carcinogenesis of the infrequent prostate cancers occurring in the transition zone, which are generally low grade (Gleason score $2-4)^{20}$ and have a considerably more bland cytonuclear appearance as compared with PIN.

\section{Clinical relevance}

In men undergoing prostate needle biopsies, PIN may be detected in conjunction with adenocarcinoma, but also as an isolated finding. The frequency of isolated PIN in prostate needle biopsies varies considerably, ${ }^{21}{ }^{22}$ and likely depends on the population being investigated (referral vs screening) and the number and quality of biopsies taken. In addition, there is substantial interobserver variation among non-genitourinary pathologists in the recognition and diagnosis of PIN. ${ }^{23}{ }^{24}$ Earlier studies reported a high detection rate of adenocarcinoma in men who underwent repeat biopsy after an initial diagnosis of
Table 1 Neoplastic processes involving prostatic ducts and their main differential diagnosis

Prostatic intraepithelial neoplasia

Atypical basal cell hyperplasia

Prostatic adenocarcinoma (acinar)

Prostatic ductal adenocarcinoma

Prostatic ductal adenocarcinome

Prostatic acinar adenocarcinoma, including pseudohyperplastic

adenocarcinoma

Prostatic invasion by rectal adenocarcinoma

Verumontanum hyperplasia

Intraductal carcinoma

Cribriform prostatic intraepithelial neoplasia (PIN)

Cribriform pattern 3 acinar adenocarcinoma

Urothelial cell carcinoma with comedonecrosis

Urothelial carcinoma (primary or secondary) Intraductal carcinoma

isolated PIN. ${ }^{21} 222526$ As a consequence, repeat prostate biopsy was generally recommended in men with the finding of isolated PIN within a period of 6 months. ${ }^{27}{ }^{28}$ More recent studies, based on large numbers of men with isolated PIN on needle biopsy, showed that the risk of prostate cancer on subsequent biopsy is not significantly increased when compared with men who had an initial benign (negative) biopsy. ${ }^{22}{ }^{25}$ The latter authors, therefore, no longer recommend early repeat biopsy, and, depending on other clinical parameters, suggest postponing repeat biopsy for 1 year. As a consequence of the new recommendations (based on the most commonly identified and more indolent forms of PIN), it follows that the identification of the less common PIN lesions, which may be associated with more aggressive carcinoma, becomes clinically relevant. $^{29}$

\section{Differential diagnosis of PIN lesions with ductal morphology} Atypical basal cell hyperplasia

As described, the diagnosis of PIN is based on the presence of dysplastic features (prominent nucleoli, enlarged nuclei and increased cytoplasmic density) within the cells of the luminal, but not the basal, compartment. In the case of atypical basal cell hyperplasia, multiple layers of basal cells with enlarged, vesicular nuclei and prominent nucleoli ${ }^{30}$ are generally covered by a single, somewhat flattened, luminal cell layer with bland nuclei. Atypical basal cell hyperplasia is a benign lesion without clinical consequence.

\section{Prostatic adenocarcinoma (conventional acinar)}

In prostate needle biopsies, tangential cuts of glandular or ductal outpouchings lined by dysplastic cells of PIN may give the false impression of invasive disease. ${ }^{31}$ By examining multiple levels to demonstrate continuity of the outpouchings with adjacent glands or ducts, and by demonstration of a basal cell layer, a diagnosis of PIN can often be made. ${ }^{32}$ Otherwise, a diagnosis of PIN with atypical glands or lesion suspicious for adenocarcinoma may be made. In the latter case, an early follow-up biopsy is recommended.

\section{Prostatic ductal adenocarcinoma}

If circumscribed ducts show distended lumena filled with neoplastic cells with a cribriform or papillary architecture, the differential diagnosis between PIN (involving a larger prostatic duct) and ductal adenocarcinoma may be considered. The diagnostic features of prostatic ductal adenocarcinoma are discussed later in this review. 

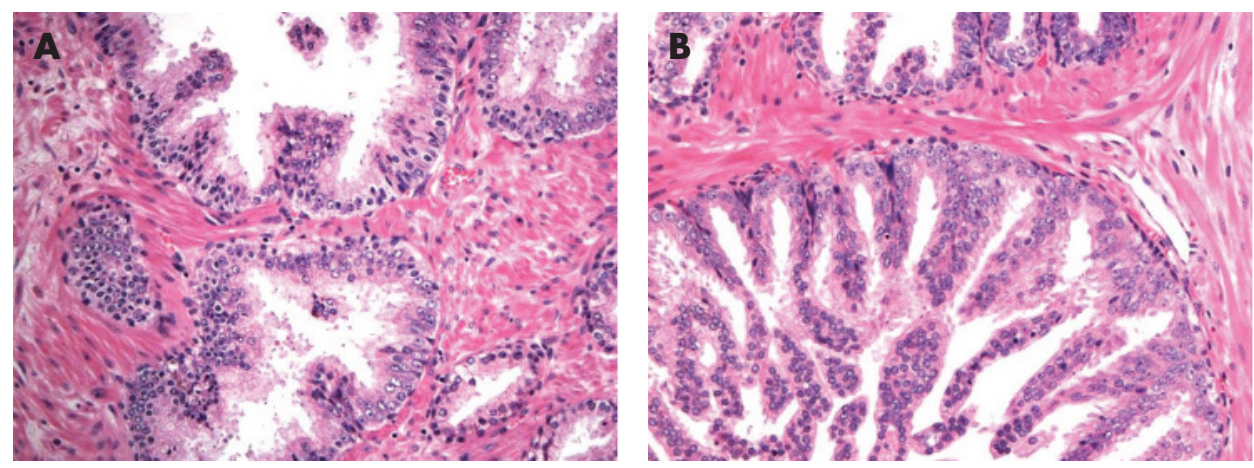

Figure 2 Some architectural patterns of PIN: (A) tufted, (B) micropapillary, (C) cribriform and (D) comedo-type PIN.
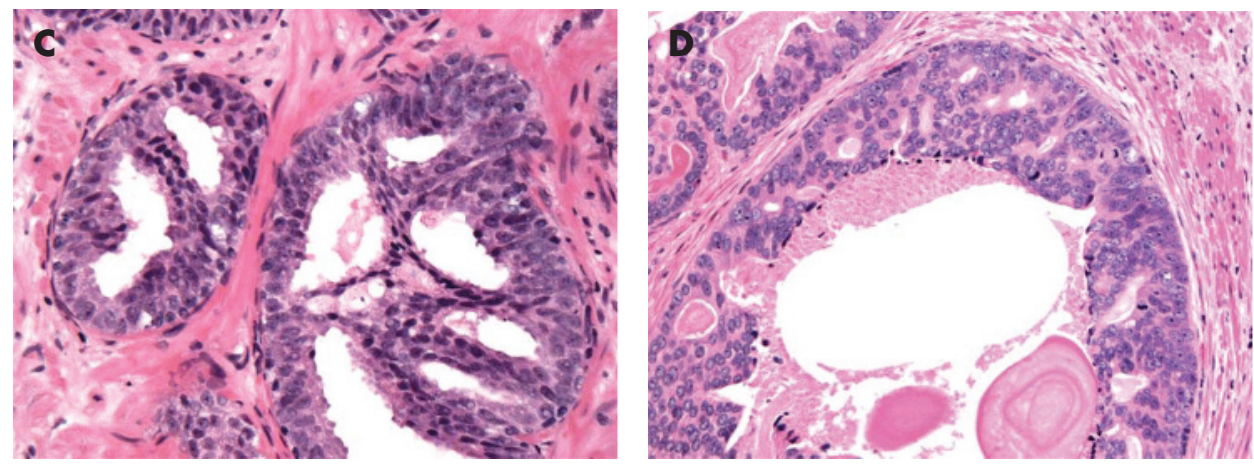

\section{Immunohistochemical features of PIN}

Almost all morphological variants of PIN share the overexpression of $\alpha$-methylacyl coenzyme A racemase/P504S (AMACR/P504S) with adenocarcinoma. As shown by immunohistochemical techniques, the overexpression of AMACR/ P504S helps to distinguish PIN in most cases from benign lesions like atrophy and atypical basal cell hyperplasia.. ${ }^{32}$ A further distinctive feature of PIN is the presence of a continuous or frequently patchy, disrupted basal cell layer. This is highlighted by staining for high-molecular-weight cytokeratins (34ßE12 or K903) or the basal cell marker p63. ${ }^{32}{ }^{33}$ In contrast, all variants of prostatic adenocarcinoma lack basal cells entirely. However, although these markers are extremely helpful (particularly in combination), they should be used cautiously and always in conjunction with conventional H\&E histological assessment. Experienced pathologists will be guided by histological features in conjunction with immunohistochemical findings, as some benign lesions may also lack basal cells or show expression of AMACR. ${ }^{32}{ }^{34}$

\section{PROSTATIC DUCTAL ADENOCARCINOMA Definition and morphology}

The rare ductal subtype of prostatic adenocarcinoma accounts for a mere $0.2-0.8 \%$ of all prostate cancers as a dominant pattern. ${ }^{35-37}$ It is frequently (in up to $3 \%$ of prostate cancer diagnoses) found as a minor component of conventional-type (acinar) adenocarcinoma. In $>80 \%$ of prostatic ductal adenocarcinomas, acinar adenocarcinoma is found within affected prostates, usually in close proximity to the ductal component. Tumour cells have abundant, frequently amphophilic, but occasionally clear, cytoplasm. Consistent with the features of large duct epithelium, the columnar neoplastic cells form a pseudostratified epithelium, often lining papillary structures with true fibrovascular cores (fig 3). ${ }^{38} 39$ Nuclei are large, mostly elongated or oval, and often contain a single macronucleolus. In some cases, there are numerous mitoses. Cytological atypia ranges from minimal to marked, the former making diagnosis particularly difficult on needle biopsy. Within a given tumour, multiple architectural patterns may be present. These range from a strikingly papillary appearance, with slender fibrovascular cores resembling endometrial carcinoma; to cribriform, making the distinction between cribriform PIN and cribriform pattern 3 carcinoma challenging; to solid, which, when circumscribed and round, is indistinguishable from solid or comedo-type Gleason pattern 5 acinar adenocarcinoma. Most ductal adenocarcinomas are equivalent to Gleason pattern 4, but, in the presence of comedo-type necrosis, they are classified as Gleason pattern 5.39 ${ }^{40}$ Occasionally, the ductal component can be comprised solely of well-circumscribed cribriform nests equivalent to Gleason pattern 3. Ductal adenocarcinoma can often be found growing within prostatic ducts (intraductal spread) with or without regional invasion. As a consequence, and further confusing accurate interpretation, a given needle core may demonstrate ductal adenocarcinoma growing along prostatic ducts with demonstrable basal cells, although this finding is (fortuitously) rare in isolation.

Prostatic ductal adenocarcinoma was once thought to be derived from the verumontanum (a müllerian duct remnant), and, because of its morphological resemblance to the endometrium, it was historically known as endometrial (or endometrioid) carcinoma. ${ }^{38-42}$ The tumour has since been shown to resemble acinar adenocarcinoma with regard to clinical behaviour, including response to orchiectomy, and ultrastructural characteristics. Further findings have disproved the postulated origin in the verumontanum and, as a consequence, the endometrial reference has been abandoned. ${ }^{42-44}$ Given their morphological and immunohistochemical similarities, ductal adenocarcinoma is thought either to derive from prostatic duct epithelium (histogenetic view) or to acquire its morphology through differentiation of acinar luminal cells into tumour cells, with the morphology of central or transition zone duct epithelium surrounding the urethra (transdifferentiation). Although controversy still remains, prostatic ductal adenocarcinoma seems now to be accepted as a pathological entity distinct from conventional (acinar) adenocarcinoma. ${ }^{45}$ 

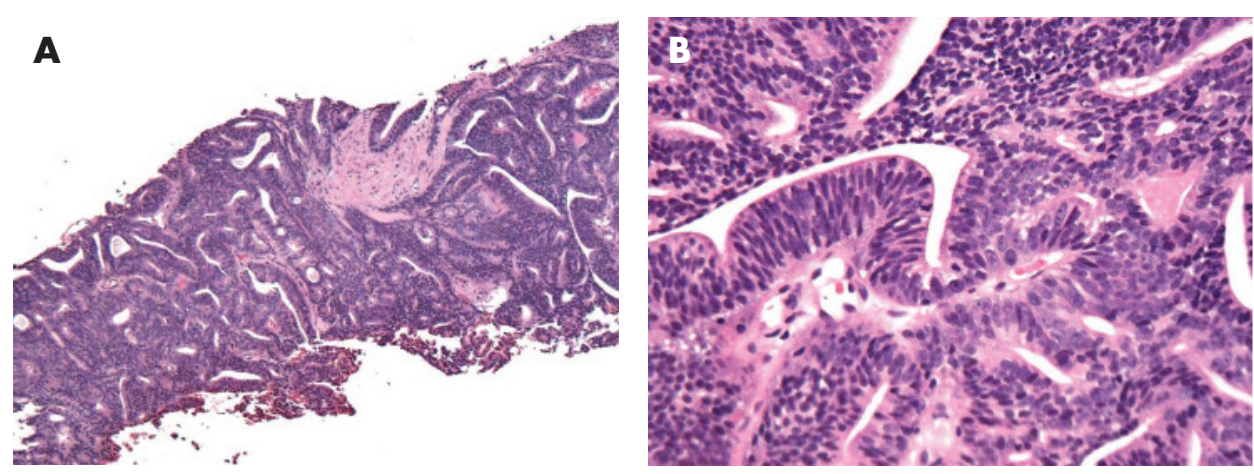

Figure 3 Core biopsy with ductal adenocarcinoma. (A) At high power (B) the lining by pseudostratified tall columnar epithelial cells can be appreciated.

\section{Architectural variants of prostatic ductal adenocarcinoma \\ Papillary variant}

The papillary pattern of ductal adenocarcinoma has a distinctive papillary architecture with papillary fronds supported by true fibrovascular cores. This is in contrast to the pseudopapillary fronds sometimes seen in micropapillary PIN, which do not contain true fibrovascular cores. ${ }^{6}$ Papillae are lined by tall columnar cells forming a single pseudostratified layer reminiscent of endometrial carcinoma. ${ }^{38}$ Although initially considered unique to prostatic ductal adenocarcinoma, conventional type acinar carcinoma has also been shown to variably display papillary architecture within peripheral foci at an incidence 10 times that of the reported incidence of ductal

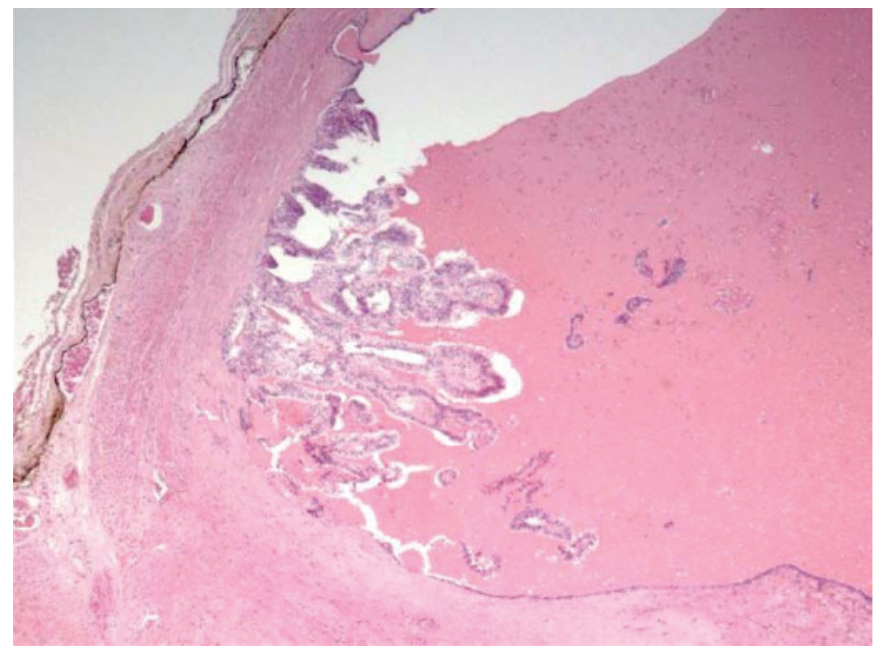

Figure 4 Cystic variant of ductal adenocarcinoma of the prostate, located in the peripheral zone. Papillary formations lined by tall columnar cells protrude in the cyst. carcinoma. ${ }^{46}$ This finding has brought under scrutiny the very existence of ductal adenocarcinoma (particularly when found peripherally), the details of which will be discussed later.

\section{Other variants}

The cribriform pattern of ductal adenocarcinoma is more commonly seen in peripheral foci, although it is certainly seen centrally. The cribriform pattern is characterised by large lumen-spanning cell masses perforated by glandular structures forming round, elongated oval and/or slit-like lumena. Recently, a number of cases of cystic ductal adenocarcinoma of the prostate have been described (fig 4), occurring both in the central and in the peripheral zones of the prostate. ${ }^{47}$ An "individual gland" pattern has also been described, characterised by single glands with a malignant-appearing pseudostratified tall columnar epithelium. ${ }^{36}$ Finally, ductal adenocarcinoma has been reported to represent the carcinomatous component of rare prostatic carcinosarcomas. ${ }^{49}$

\section{Clinical relevance}

When symptomatic, ductal adenocarcinoma most commonly presents with urinary obstruction and/or haematuria, as a consequence of the friable exophytic papillary lesion that it commonly forms within the prostatic urethra. Accordingly, ductal adenocarcinoma is frequently diagnosed at transurethral resection or visualised at the time of cystoscopy. Needle biopsies, which preferentially sample the peripheral zone of the gland, have historically yielded low rates of ductal adenocarcinoma. Although it is thought to be related to its more central location, ductal adenocarcinoma (when it represents the dominant lesional morphology) is less reliably flagged by digital rectal examination screening strategies, and a predictive correlation with serum PSA levels has proved difficult to ascertain. It has been postulated that the unpredictable PSA correlation results from the readily available prostatic duct excretion pathway rather than attenuated production of PSA by the tumour cells. Anecdotally, advanced cases of ductal adenocarcinoma have been found to correlate

Table 2 Histopathological criteria to distinguish prostatic lesions with ductal morphology

\begin{tabular}{llll}
\hline Morphological criteria & PIN & Intraductal carcinoma & Ductal carcinoma \\
\hline Size of glands/ducts & Normal-distended & Markedly distended & Large duct-like structures \\
Lumen spanning & Occasional & Required & Yes \\
Basal cells & Yes & Yes, disrupted & Rarely \\
Columnar & No & Occasionally & Yes \\
Papillary structures & No & No & Occasionally \\
Cribriform pattern & Yes & Frequently & Frequently \\
Central necrosis & No & Occasionally & Occasionally \\
Severe nuclear atypia & Rare & Occasionally & Occasionally \\
Maturation effect & Yes & Occasionally & No \\
Stromal haemosiderin & No & No & Occasionally \\
\hline
\end{tabular}

PIN, prostatic intraepithelial neoplasm. 


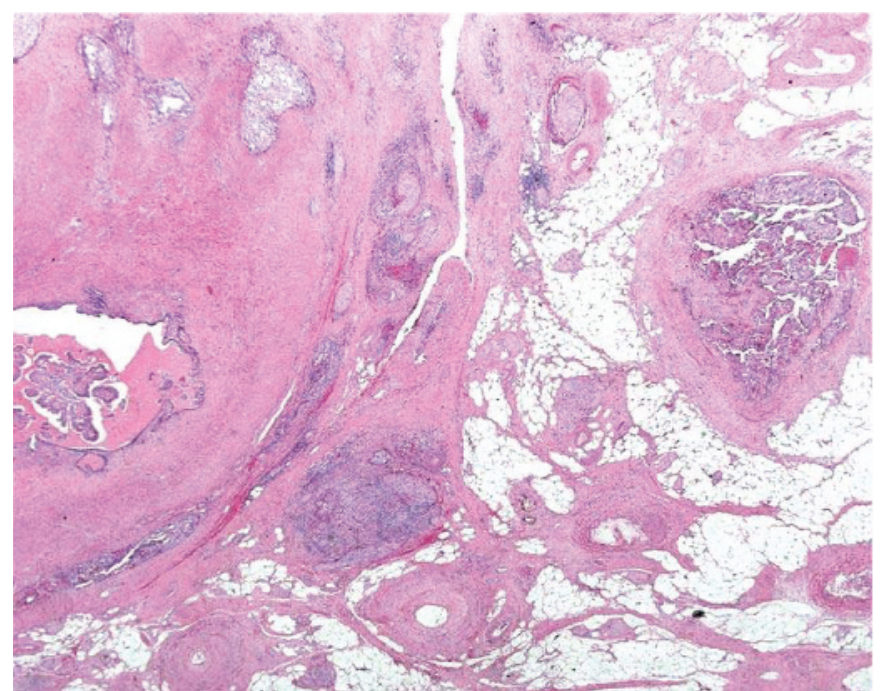

Figure 5 Focus of ductal adenocarcinoma located in the extraprostatic adipose tissue.

with elevated serum PSA. ${ }^{39}$ With the dramatic increase in prostate needle biopsies after the advent of PSA screening, and even more so with the initiation of extensive sampling $(>10$ cores) at low PSA levels, ductal adenocarcinoma is nowadays likely to be diagnosed on needle biopsy specimens more frequently. At the University Health Network, Toronto, Ontario, Canada, of the nine cases of ductal carcinoma between 2002 and 2005, four were identified at needle biopsy. When diagnosed in needle biopsies, it is more often associated with an unfavourable prognosis, as reflected in its Gleason grade $4 .{ }^{39}$

\section{THE CONTROVERSY}

The nomenclature of focal ductal adenocarcinoma confined to the peripheral zone is controversial, and, given the tumour's resemblance to acinar adenocarcinoma with ductal (papillary and cribriform) features and the frequent coexistence of acinar and ductal morphology within prostate cancers, some pathologists hold firm that a central location is the only specific criterion for diagnosis of ductal adenocarcinoma. Furthermore, the central location is associated with its distinct clinical symptomatology (late rise in serum PSA, urinary obstruction, negative digital rectal examination). ${ }^{46}$ Thus, inclusion of peripheral zone prostate cancers with ductal morphology within the entity of "ductal adenocarcinomas" would eliminate the practicality of subcategorisation. In our opinion, and others, ${ }^{50}$ ductal carcinomas should be considered a mere morphological variant of prostatic adenocarcinoma, and not as a separate entity. Further substantiating this opinion, foci of carcinoma with papillary features are frequently found at the periphery of the prostate and even in prostate cancer foci within extraprostatic adipose tissue (fig 5). Ductal morphology may simply be a consequence of the availability of space for exuberant papillary growth. A consensus has not yet been reached with regard to the reporting of isolated foci of carcinoma with ductal morphology in the peripheral zone. We believe that the clinical relevance of subcategorisation of such peripheral foci is moot, as these lesions are designated high grade using currently accepted Gleason grading criteria, and evidence to support alternate treatment or follow-up strategies does not exist.

\section{Differential diagnosis}

\section{Conventional-type (acinar) adenocarcinoma}

The distinguishing feature of ductal adenocarcinoma is the finding of circumscribed papillary and/or cribriform duct-like structures lined by tall columnar cells with ample cytoplasm (table 2). ${ }^{39}$ Some ductal adenocarcinomas show extensive fibrosis and heavy deposits of haemosiderin pigment-an observation that is extremely rare in pure acinar adenocarcinomas. ${ }^{39}$ It may also be problematic to separate the pseudohyperplastic variant of prostatic adenocarcinoma ${ }^{51}$ from ductal carcinoma, as the neoplastic cells in the pseudohyperplastic variant of adenocarcinoma also have a columnar appearance with abundant cytoplasm. However, true papillary structures and duct-like structures are not found in the latter variant. Immunohistochemical markers are not useful for this differential diagnosis.

\section{Rectal carcinoma}

The identification of a prostate-localised carcinoma characterised by tall columnar cells should always raise the suspicion of direct extension of rectal carcinoma with prostatic involvement. The presence of mucin, the absence of PSA and PAP immunostaining, in addition to the generally intense and diffuse expression of carcinoembryonic antigen (CEA) and caudal type homeobox transcription factor 2 (CDX2) by rectal carcinoma, should permit the straightforward distinction of these two histologically similar malignancies. ${ }^{52} 53$

\section{Verumontanum hyperplasia}

Some ductal adenocarcinomas may show minimal cytonuclear atypia. When such a lesion is encountered at prostate needle biopsy, it may be difficult to separate this lesion from hyperplasia of the verumontanum (fig 6), particularly when associated with papillary formations. ${ }^{54} \mathrm{~A}$ combination of immunohistochemistry using basal cell markers and H\&E cytonuclear features may be helpful, because verumontanum hyperplasia, a benign entity, shows continuous basal cell staining and bland nuclear features. Careful examination at multiple levels may be required for accurate differentiation. AMACR immunostaining in ductal adenocarcinoma (and an absence of staining in verumontanum hyperplasia) may also help to delineate the two.

\section{Immunohistochemistry}

Currently, no specific marker exists to separate ductal adenocarcinomas from conventional acinar adenocarcinoma. In virtually all cases, the neoplastic cells of ductal adenocarcinoma are at least focally PSA and PAP positive. ${ }^{36}$ Tumour spread frequently occurs through existing ducts, with or without stromal invasion, and, as a result, some cases of obvious ductal adenocarcinoma can have residual basal cells with continuous or interrupted staining by high molecular weight cytokeratin or p63. ${ }^{55}$ The proliferation associated marker Ki-67 has been used by some authors to differentiate ductal adenocarcinoma from PIN; they claim that ductal adenocarcinomas have a much higher proliferative rate than (lumen spanning) cribriform or papillary PIN with no overlap. ${ }^{56}$ Immunohistochemical staining with $\alpha$-methylacyl-CoA racemase/P504S (AMACR/P504S) staining is comparable to other forms of prostatic adenocarcinoma, and is of no use in differentiating ductal adenocarcinomas from PIN or conventional (acinar) adenocarcinoma. ${ }^{32}$

\section{INTRADUCTAL CARCINOMA Definition and morphology}

Perhaps more controversial than the concept of ductal adenocarcinoma is the "entity" known as intraductal carcinoma, which is presented as a unifying theory to explain the morphologically malignant lumen-spanning lesions within prostatic ducts and acini of prostatic epithelial origin, including prostatic ductal adenocarcinoma. ${ }^{8}{ }^{57}$ Morphologically, intraductal carcinoma is defined as well-circumscribed lesions bound by 


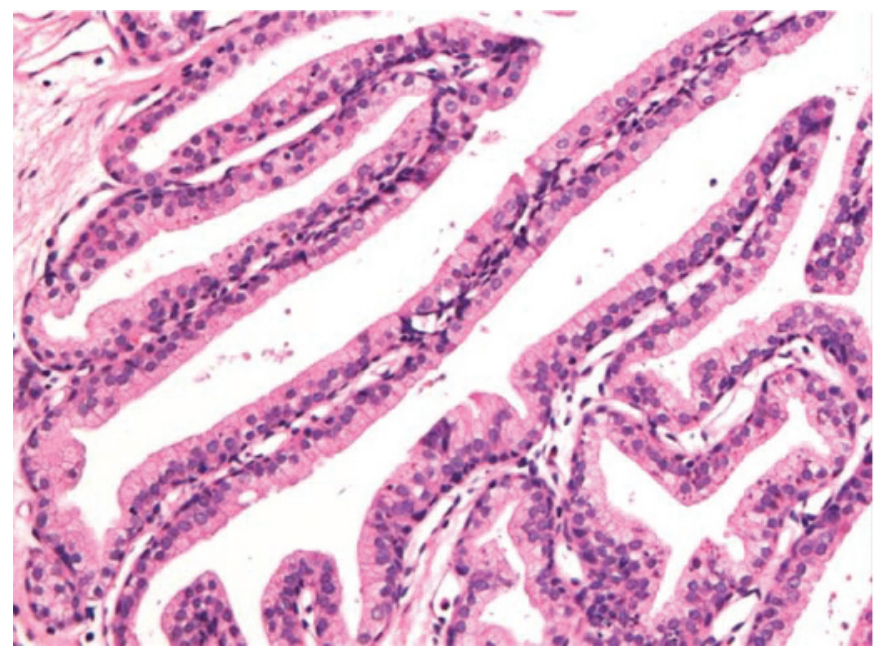

Figure 6 Verumontanum with papillary structures, lined by an outer layer of basal cells and an inner layer of columnar cells.

an intact basal cell layer (figs 7 and 8) distended by overtly malignant-appearing epithelial populations. These lumenspanning lesions are found almost exclusively in close proximity to invasive cancer (table 2 ). ${ }^{9}$ On the other hand, the more common architectural variants of PIN (tufted and micropapillary) are frequently multifocal, occurring commonly in isolation or at a distance from invasive carcinoma.

McNeal and Yemoto 9 described two separate compartments within the luminal masses constituting intraductal carcinoma: a perimeter compartment containing epithelial cells forming a ring approximating the periglandular stroma, and a central compartment. It is postulated that the central compartment represents a distinct population of cells that have attained the ability to survive without requiring intimate stromal-epithelial interactions. This is analogous to Gleason patterns $4 / 5$ carcinoma, which (cribriform Gleason pattern 3 aside) are the sole architectural patterns found in malignant foci-containing cells bound by other malignant cells without stromal contact. The perimeter compartment, arguably indistinguishable from those found in high-grade PIN, always displays prominent cytonuclear abnormalities, whereas the neoplastic cells of the central compartment are cytologically different, and are often bland in appearance. Three patterns of intraductal carcinomas have been described, predominantly based on the architecture of the inner cell masses, including those with trabecular or cribriform architecture (fig 9), and those composed of solid masses of neoplastic cells (fig 10). In both the trabecular and cribriform patterns, the neoplastic cells in the central compartment may show a putative "maturation" effect. This "maturation" effect is characterised by a reduction in nuclear size and cytonuclear atypia as compared with the cells in the perimeter. The solid pattern is characterised by a solid intraductal or intraacinar cell plug. In this pattern, the demarcation of the perimeter cells is lost, and a higher degree of cytonuclear atypia (enlargement and pleomorphism) is generally encountered. The solid pattern also commonly gives rise to central comedo-type necrosis. Comedo-type necrosis is occasionally also seen in the cribriform pattern of intraductal carcinoma.

According to some authors, intraductal carcinoma may differ from PIN in its limited response to androgen deprivation. Whereas PIN shows loss of cytonuclear atypia, including the disappearance of prominent nucleoli, ${ }^{58}$ after androgen deprivation, Montironi et al ${ }^{59}$ reported that lesions with the morphology of intraductal carcinoma are refractory to this effect.

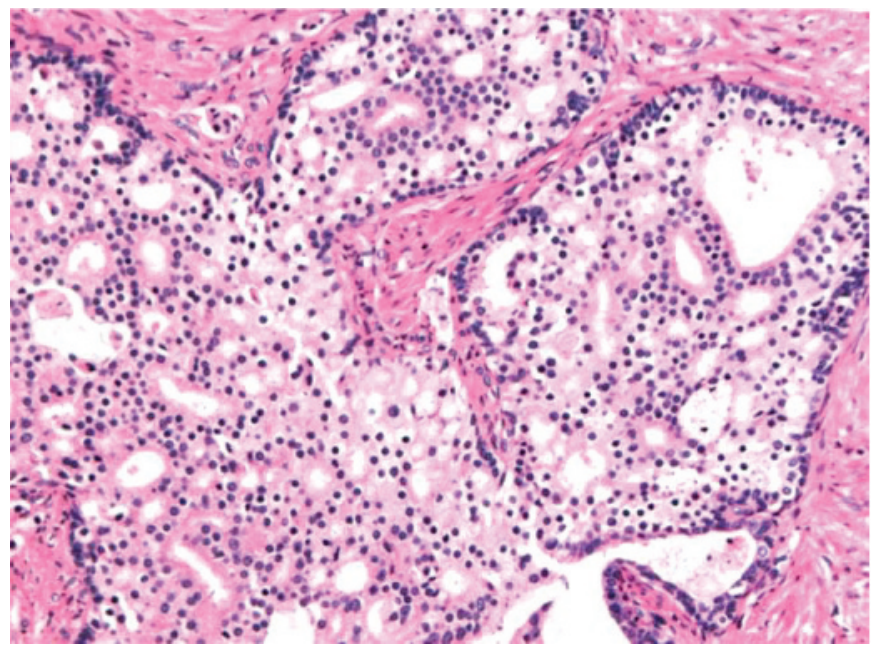

Figure 7 Lesion with ductal morphology, distended by a cribriform mass of dysplastic cells.

\section{Relationship with PIN and invasive carcinoma}

Based on the cytological and histological findings showing similarities in intraductal cell masses and the surrounding invasive components, McNeal and Yemoto ${ }^{9}$ have postulated that intraductal carcinoma represents the intraductal spread of frankly invasive carcinoma. The ability to survive within prostatic ducts and without stromal-epithelial interactions enables the tumour to involve and traverse the length of prostatic ducts, thereby resulting in rapid growth and diffuse prostatic involvement. Another compelling argument cited by these authors was the nearly exclusive localisation of intraductal carcinoma within and in the near vicinity of the overtly invasive carcinoma. This view is further substantiated by the published prognostic significance of intraductal carcinoma, most notably the reduction in time to progression after radical prostatectomy. ${ }^{29}$ 60-62

It has also been suggested that intraductal carcinoma could represent a progression from PIN, acquiring the ability to span the lumen, and, hence, growth along duct lumena.. This hypothesis is supported by the finding of isolated intraductal foci in close proximity to PIN, in addition to the cytological similarities with PIN, seen within the perimeter component of

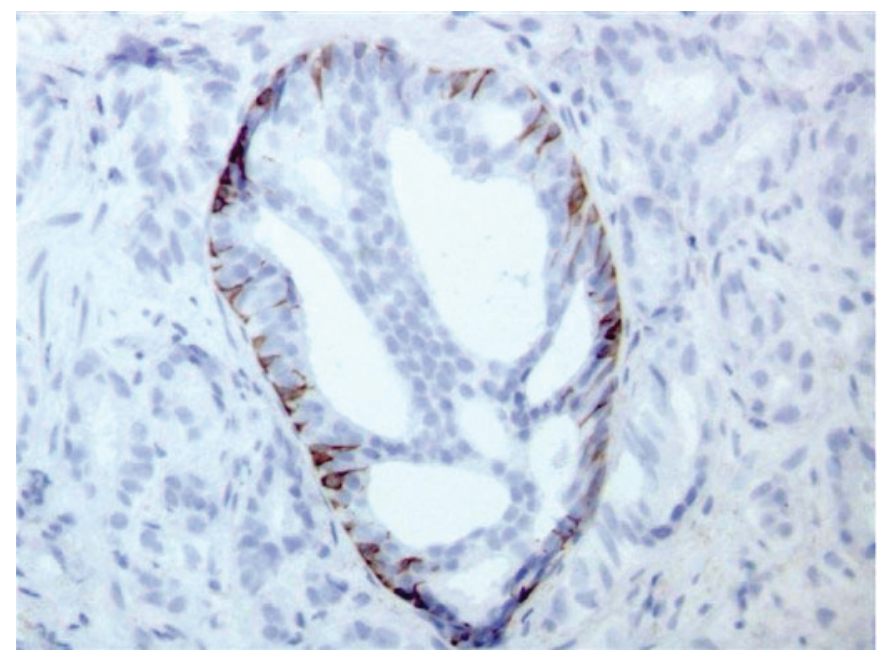

Figure 8 Immunohistochemical staining for basal cells (high molecular weight cytokeratin), depicting the outer contour of the distended duct filled with a cribriform mass of dysplastic cells. 


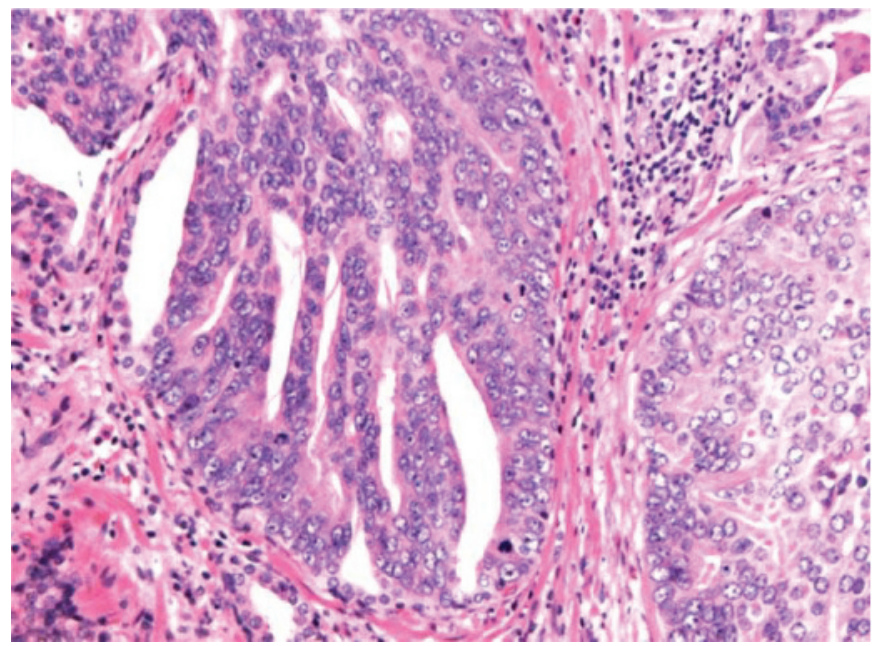

Figure 9 Distended trabecular lesion with ductal morphology, the lumen of which is largely comprised of neoplastic cells. Note the absence of maturation of the central, largely solid component. An outer rim composed of basal cells is present.

intraductal carcinoma. Furthermore, patterns of immunohistochemical staining for the androgen receptor within PIN are mirrored by the perimeter component of intraductal carcinoma. ${ }^{63}$

In their 2000 paper on the distinction between intraductal carcinoma of the prostate, high-grade PIN and invasive carcinoma, Dawkins et $a l^{64}$ describe the molecular characteristics of intraductal carcinoma foci discordant from both highgrade PIN and invasive carcinoma. This was shown by quantifying loss of heterozygosity for 12 polymorphic microsatellite markers, known to be frequently lost in prostate cancer, within foci of PIN, intraductal carcinoma, and Gleason pattern 3 and 4 invasive carcinomas. For the purpose of their study, cribriform Gleason pattern 3 carcinoma and cribriform PIN were considered to be cribriform pattern intraductal carcinoma. The findings demonstrated allelic loss in $60 \%$ of intraductal carcinoma foci, in $29 \%$ of Gleason pattern 4 cancers, in $0 \%$ of Gleason pattern 3 cancers and in $9 \%$ of PIN foci. Further complicating their conclusions, specific loci of allelic loss were dissimilar between foci of Gleason pattern 4 carcinoma and intraductal carcinoma within the same specimen. These findings suggest that intraductal carcinoma is distinct or at least far removed from PIN, and, even more puzzling, that intraductal carcinoma may represent a population of cells distinct from Gleason pattern 4 carcinoma, implying a de novo origin of this lesion. Nevertheless, the study does provide molecular evidence to separate intraductal carcinoma from the more common patterns of PIN, in that the former are more similar to Gleason pattern 4 carcinomas with respect to their extent of allelic loss.

\section{Clinical relevance}

Supporting the earlier findings of McNeal and Yemoto, ${ }^{9}$ Wilcox et al and Rubin et al ${ }^{61}{ }^{62}$ showed high-grade cribriform PIN (meeting the criteria by McNeal and Yemoto for diagnosis of intraductal carcinoma) to be an independent predictor of poor prognosis with a reduction in time to, and increased frequency of, disease progression after radical prostatectomy. They also showed an association with increased cancer volume and tumours of higher Gleason score. Notably, these studies advocating intraductal carcinoma as an entity separate from PIN are all based on prostatectomy data, and have as such been subject to criticism with respect to clinical bias. Adversaries of this view consider it impossible to reliably diagnose intraductal

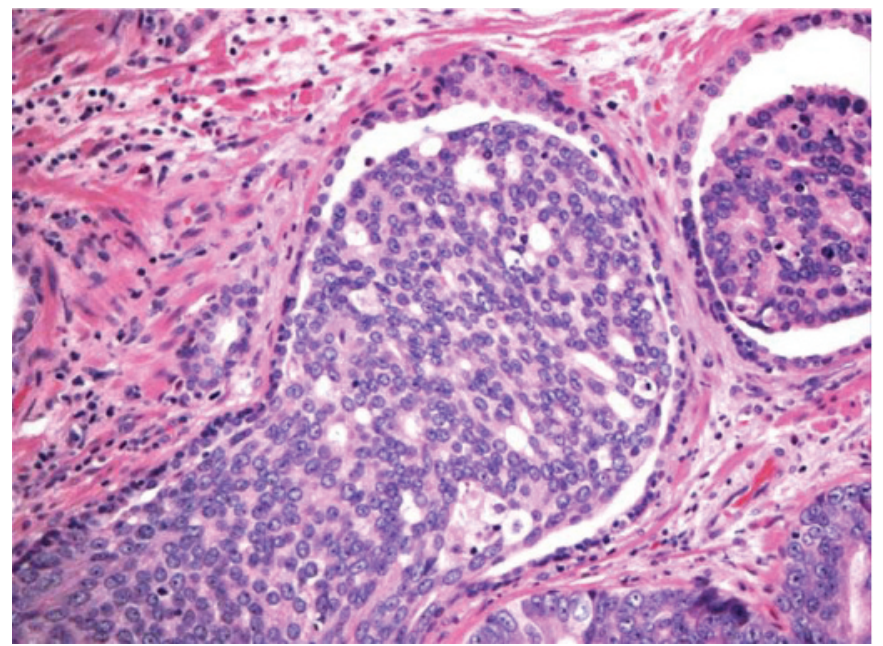

Figure 10 Distended duct, filled with a mass of dysplastic cells. The optically empty space surrounding the dysplastic cell mass is suggestive of intraductal spread.

carcinoma, and argue that its diagnosis would not result in altered patient management. A recent paper on 27 isolated cases of intraductal carcinoma diagnosed at needle biopsies has profoundly challenged this view. ${ }^{65}$ These authors formulated a number of criteria to distinguish conventional PIN from intraductal carcinoma; in the six cases treated by prostatectomy, a high-grade prostate cancer was detected, whereas three of the patients not treated by prostatectomy developed bone metastases.

As mentioned earlier, the recommendations for the follow-up management of isolated PIN have changed recently, 22 lengthening the time to repeat biopsy to 1 year. This effectively increases the importance of reporting intraductal carcinoma as an entity distinct from PIN, lest men with evidence of invasive carcinoma be permitted to progress during the time to followup. Diagnosis of "intraductal carcinoma" on a needle biopsy in the absence of an associated invasive component in the same set of biopsies is believed by some pathologists to be invariably associated with invasive disease elsewhere in the prostate. ${ }^{65}$ This notion brings into question whether patients with a diagnosis of intraductal carcinoma should be subject to repeat biopsy to prove the existence of invasive disease. A repeat

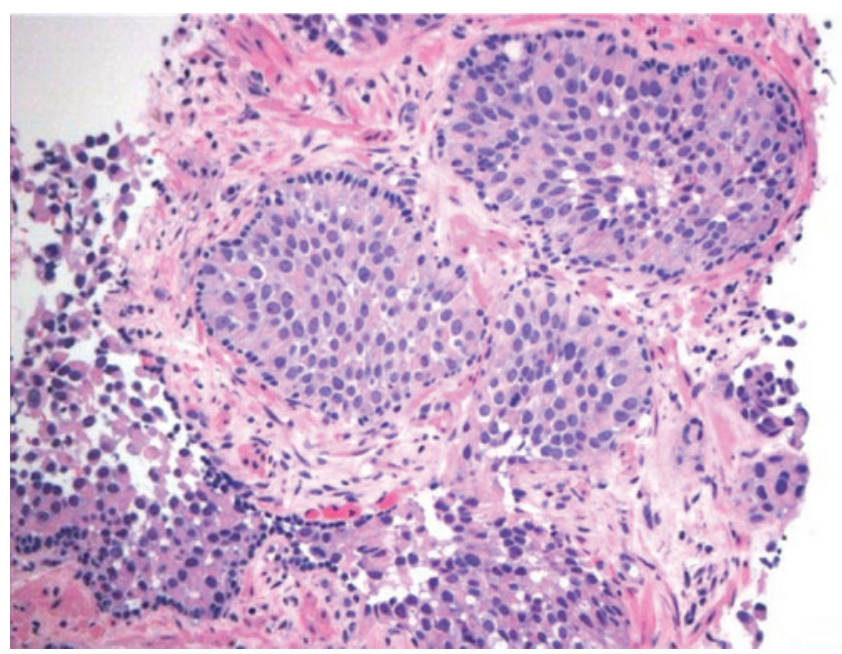

Figure 11 Prostate needle biopsy with distended ducts filled with atypical cells with urothelial morphology, representing the intraductal spread of a urothelial cell carcinoma. A basal cell layer forms the outer rim of the duct. 
Table 3 The spectrum of lesions with a cribriform appearance

\begin{tabular}{|c|c|c|c|c|}
\hline Lesion & $\begin{array}{l}\text { Basal } \\
\text { cells }\end{array}$ & $\begin{array}{l}\text { Cytonuclear } \\
\text { features }\end{array}$ & $\begin{array}{l}\text { Distension of } \\
\text { duct }\end{array}$ & Outline of duct \\
\hline Hyperplasia & Present & Bland & $\begin{array}{l}\text { None or } \\
\text { slight }\end{array}$ & Smooth \\
\hline H-PIN & Present & Nucleoli & $\begin{array}{l}\text { None or } \\
\text { slight }\end{array}$ & Smooth \\
\hline $\begin{array}{l}\text { Intraductal } \\
\text { carcinoma }\end{array}$ & Present & Nucleoli & Distended & Smooth \\
\hline $\begin{array}{l}\text { Grade } 3 \\
\text { adenocarcinoma }\end{array}$ & Absent & Nucleoli & Slight & Smooth \\
\hline $\begin{array}{l}\text { Grade } 4 \\
\text { adenocarcinoma }\end{array}$ & Absent & Nucleoli & Distended & $\begin{array}{l}\text { Irregular, or } \\
\text { outpouchings }\end{array}$ \\
\hline $\begin{array}{l}\text { Ductal } \\
\text { adenocarcinoma }\end{array}$ & $\begin{array}{l}\text { Absent, } \\
\text { rare }\end{array}$ & $\begin{array}{l}\text { Nucleoli, tall } \\
\text { columnar cells }\end{array}$ & Distended & Offen smooth \\
\hline
\end{tabular}

H-PIN, high-grade prostatic intraepithelial neoplasm.

biopsy to provide a definite diagnosis of invasive disease may thus not be necessary if the stringent criteria for "intraductal carcinoma" are fully met. These criteria are defined as the presence of at least one lumen-spanning lesion with substantial duct distension showing severe nuclear atypia involving the neoplastic cells in the centre of the duct. The realists among us, however, given the vanishingly rare frequency of such stringent findings isolated from frankly invasive disease, realise that the invasive nature of repeat biopsy as compared with radical prostatectomy favours strongly the former. These authors submit that stringent criteria should be relaxed somewhat in favour of designating all patients with lesions suspicious for intraductal carcinoma as candidates for early re-biopsy. In the rare event that intraductal carcinoma is encountered in a prostate biopsy with a Gleason pattern 3 adenocarcinoma as the only invasive component, it should be considered that this generally indicates the presence of associated high-grade (Gleason pattern 4 or 5) carcinoma elsewhere within the prostate. We recommend that the pathologist should add a comment to the report mentioning that the presence of intraductal carcinoma is invariably associated with a highgrade carcinoma component. Upgrading a prostate cancer with unequivocal comedo-type necrosis within a focus of intraductal

\section{Take-home messages}

- The view that ductal adenocarcinoma represents a separate category of prostatic adenocarcinoma is not supported by strong morphological, molecular or clinical evidence. This warrants grouping ductal adenocarcinomas with (conventional-type) acinar adenocarcinomas.

- Using stringent morphological criteria, intraductal carcinoma can be distinguished in needle biopsies from the other architectural variants of prostatic intraepithelial neoplasia of less clinical significance (table 2).

- The (rare) finding of isolated intraductal carcinoma in needle biopsies should prompt early repeat biopsy and/ or treatment.

- When intraductal carcinoma is found in conjunction with a Gleason pattern 3 carcinoma, a comment should be added to the pathological report stating that intraductal carcinoma is invariably associated with high-grade (Gleason pattern 4 or 5) adenocarcinoma. carcinoma with concomitant Gleason pattern 3 carcinoma to a Gleason score $8(3+5)$ adenocarcinoma might be considered.

A recent study polled subspecialty genitourinary pathologists on their reporting practices with respect to lesions fitting the above-stated criteria for intraductal carcinoma. ${ }^{66}$ In all, $44 \%$ of pathologists polled currently reported intraductal carcinoma; $27 \%$ reported PIN with atypical features and 20\% simply reported said lesions as PIN. One wonders what the urologist would infer from a diagnosis "PIN with atypical features"? It is obvious that clear guidelines are needed for reporting lesions with this morphology.

\section{Differential diagnosis \\ Cribriform lesions}

Cribriform lesions of the prostate encompass a spectrum from entirely benign to high-grade malignancy. Table 3 outlines the differential diagnostic criteria of each of these cribriform lesions. Current guidelines stipulate that lumen-spanning cribriform lesions with demonstrable basal cell layers are designated PIN. Cribriform PIN represents about $5 \%$ of isolated PIN found in biopsies of asymptomatic men, ${ }^{22}{ }^{26}$ and PIN with this architectural pattern was not found by all authors to be related to an increased risk of subsequent prostate cancer. ${ }^{22}{ }^{25}$ It remains to be seen whether (within the set of cribriform PIN) a subset of lesions that represent intraductal carcinoma- for example, those with substantial luminal distension and/or substantial cytonuclear atypia-can be identified with sufficient reliability. As yet, no specific markers exist to solve this issue, and it might therefore be recommended to report this kind of lesion as intraductal carcinoma (table 2). In our opinion, lesions with identifiable central comedo-type necrosis and/or neoplastic cells with clear-cut nuclear atypia present within the central compartment of distended ducts lined by scattered basal cells should not simply be reported as PIN, but as intraductal carcinoma (figs 9 and 10). The diagnosis of the rather uncommon pure variant of cribriform adenocarcinoma of small size observed in a needle biopsy specimen requires a demonstrable absence of a basal cell layer using immunohistochemical markers like p63 and/or high-molecular-weight cytokeratin.

\section{Ductal adenocarcinoma}

Rarely, lesions with ductal morphology, comprised of tall columnar cells with severe cytonuclear atypia, may be encountered, with occasional scattered basal cells in their lining. This finding will prompt a differential diagnosis between ductal adenocarcinoma and intraductal carcinoma. In this case, it is justified to make a diagnosis of ductal adenocarcinoma. If, however, a relatively continuous lining of basal cells is present, a certain degree of reluctance is understandable in making a definitive diagnosis of invasive carcinoma. Clinicians should be warned of the high likelihood of adjacent invasive disease, and repeat biopsy targeting the area in question is strongly recommended.

\section{Immunohistochemistry and molecular markers}

The same markers that can be used to distinguish PIN from benign glands and ducts on one hand from adenocarcinoma on the other also apply to intraductal carcinoma. Recently, a translocation involving the androgen-regulated transmembrane proteinase, serine (TMPRSS) gene and the transcription factors ets and erg was reported to occur in the majority of prostatic adenocarcinoma, but only in a small proportion of PIN. ${ }^{67}{ }^{68}$ If the results of these recent findings hold true, the demonstration of a cancer-specific translocation may represent the first genetic marker able to help distinguish PIN from intraductal adenocarcinoma. 


\section{UROTHELIAL CELL CARCINOMA INVOLVING PROSTATIC DUCTS (PRIMARY OR SECONDARY) Definition and morphology}

Primary urothelial cell carcinoma of the prostate may arise from the prostatic urethra or from the urothelial lining of the larger periurethral prostatic ducts. More commonly, secondary involvement of prostatic ducts by urothelial carcinoma of the bladder is the consequence of propagation of carcinoma in situ by pagetoid spread. ${ }^{69}$ Urothelial cell carcinomas involving the prostate are most often diagnosed in transurethral resections of the prostate, and occasionally in prostate needle biopsies (fig 11). ${ }^{70}$ Because most cases represent high-grade (nonpapillary) carcinomas, they show the morphology of typical carcinoma in situ of the bladder-that is, severe nuclear changes with hyperchromasia and anisonucleosis. They may also show squamous differentiation. Tumour cells may fill the lumen of the prostatic ducts and central necrosis may occur, giving the picture of comedo-type necrosis. ${ }^{70}$ Occasionally, pagetoid spread of neoplastic urothelial cells within the prostatic ducts may be identified. Pagetoid spread is seen as atypical cells (either single or in small nests) interspersed within the benign epithelial lining of the prostatic ducts.

\section{Clinical relevance}

If involvement of prostatic ducts by urothelial cell carcinoma is found on prostate needle biopsy, the primary origin of the cancer within the urinary bladder and/or urethra needs to be established. The demonstration of prostatic stromal invasion by urothelial carcinoma cells carries an unfavourable prognosis.

\section{Immunohistochemistry}

Urothelial cell carcinoma shows an intense and diffuse expression of cytokeratin 7 and, to a lesser extent, p63, but not of PSA or PAP. This should allow their distinction from neoplastic lesions of prostatic origin in virtually all cases. ${ }^{71}$

\section{Authors' affiliations}

M Pickup, T H Van der Kwast, Department of Pathology, Princess Margaret Hospital, University Health Network, Toronto, Ontario, Canada

Competing interests: None declared.

\section{REFERENCES}

1 McNeal JE. Normal histology of the prostate. Am J Surg Pathol 1988;2:619-33.

2 Abrahamsson PA, Lilja H, Falkmer S, et al. Immunohistochemical distribution of the three predominant secretory proteins in the parenchyma of hyperplastic and neoplastic prostate glands. Prostate 1988;12:39-46.

$3 \mathrm{McNeal} \mathrm{JE}$, Reese JH, Redwine EA, et al. Cribriform adenocarcinoma of the prostate. Cancer 1986;58:1714-19.

4 Bostwick DG, Brawer MK. Prostatic intra-epithelial neoplasia and early invasion in prostate cancer. Cancer 1987;59:788-94.

5 Bostwick DG, Qian J. High-grade prostatic intraepithelial neoplasia. Mod Pathol 2004; 17:360-79.

6 Bostwick D, Amin M, Dundore P, et al. Architectural patterns of high-grade prostatic intra-epithelial neoplasia. Hum Pathol 1993;24:298-310.

7 Jackson MA, Heshmat MY. Ductal spread in prostatic carcinoma. Cancer 1985;56:1566-73.

8 Kovi J, Jackson MA, Heshmat MY. Ductal spread in prostatic carcinoma. Cancer 1985;56:1566-73.

9 McNeal JE, Yemoto CE. Spread of adenocarcinoma within prostatic ducts and acini: morphologic and clinical correlations. Am J Surg Pathol 1996;20:802-14.

10 Reyes AO, Swanson PE, Carbone JM, et al. Unusual histologic types of highgrade prostatic intraepithelial neoplasia. Am J Surg Pathol 1997;21:1215-22.

11 Berman DM, Yang J, Epstein JI. Foamy gland high-grade prostatic intraepithelial neoplasia. Am J Surg Pathol 2000;24:140-4.

12 Bostwick DG. Prospective origins of prostate carcinoma: prostatic intraepithelial neoplasia and atypical adeomatous hyperplasia. Cancer 1996;78:330-6.

13 Bostwick DG, Montironi R, Sesternenn IA. Diagnosis of prostatic intra-epithelial neoplasia: prostate working group/consensus report. Scand I Urol Nephrol Suppl 2000;205:3-10.

14 Foster CS, Bostwick DG, Bonkhoff $\mathrm{H}$, et al. Cellular and molecular pathology of prostate cancer precursors. Scand J Urol Nephrol Suppl 2000;205:19-43.

15 Qian J, Wollan P, Bostwick DG. The extent and multicentricity of high-grade prostatic intra-epithelial neoplasia in clinically localized prostatic adenocarcinoma. Hum Pathol 1997;28:143-8.
16 Montironi R, Mazzucchelli R, Santinelli A, et al. Incidentally detected prostate cancer in cystoprostatectomies: pathological and morphometric comparison with clinically detected cancer in totally embedded specimens. Hum Pathol 2005; 36:646-54.

17 Emmert-Buck MR, Vocke CD, Pozzatti RO, et al. Allelic loss on chromosome 8p12-21 in microdissected prostatic intraepithelial neoplasia. Cancer Res 1995;55:2959-62.

18 Qian J, Jenkins RB, Bostwick DG. Genetic and chromosomal alterations in prostatic intraepithelial neoplasia and carcinoma detected by fluorescence in situ hybridization. Eur Urol 1999;35:479-83.

19 Ashida S, Nakagawa H, Katagiri T, et al. Molecular features of the transition from prostatic intraepithelial neoplasia (PIN) to prostate cancer: genome-wide gene-expression profiles of prostate cancers and PINs. Cancer Res 2004;64:5963-72.

20 Harvei S, Skjorten FJ, Robsahm TE, et al. Is prostatic intraepithelial neoplasia in the transition/central zone a true precursor of cancer? A long-term retrospective study in Norway. Br J Cancer 1998;78:46-9.

21 Hoedemaeker RF, Kranse R, Rietbergen JB, et al. Evaluation of prostate needle biopsies in a population-based screening study: the impact of borderline lesions. Cancer 1999;85:145-52.

22 Epstein JI, Herawi M. Prostate needle biopsies containing prostatic intraepithelial neoplasia or atypical foci suspicious for carcinoma: implications for patient care. J Urol 2006;175:820-34.

23 Epstein JI, Grignon DJ, Humphrey PA, et al. Interobserver reproducibility in the diagnosis of prostatic intraepithelial neoplasia. Am J Surg Pathol 1995; 19:873-86

24 Allan CK, Bostwick DG, Hayes JA, et al. Interobserver variability in the diagnosis of high-grade prostatic intraepithelial neoplasia and adenocarcinoma. Mod Pathol 1996;9:742-51.

25 Vis AN, Hoedemaeker RF, Roobol M, et al. The predictive value for prostate cancer of lesions that raise suspicion of concomitant carcinoma. Cancer $2001 ; 92: 524-34$.

26 Kronz JD, Shaikh AA, Epstein Jl. High-grade prostatic intraepithelial neoplasia with adjacent small atypical glands on prostate biopsy. Hum Pathol 2001;32:389-95.

27 Bostwick DG, Qian J, Frankel K. The incidence of high grade prostatic intraepithelial neoplasia in needle biopsies. J Urol 1995;154:1791-4.

28 Bostwick DG. Prostatic intraepithelial neoplasia is a risk factor for cancer. Semin Urol Oncol 1999;17:187-98.

29 Cohen R, Chan W, Edgar S, et al. Prediction of pathologic stage and clinical outcome in prostate cancer; an improved preoperative sequential model incorporating biopsy determined intraductal carcinoma. Br J Urol 1998;81:413-18.

30 Epstein JI, Armas OA. Atypical basal cell hyperplasia of the prostate. Am J Surg Pathol 1992;16:1205-14.

31 Kronz JD, Allan $\mathrm{CH}$, Shaikh AA, et al. Predicting cancer following a diagnosis of high-grade prostatic intraepithelial neoplasia on needle biopsy: data on men with more than one follow-up biopsy. Am J Surg Pathol 2001;8:1079-85.

32 Hameed O, Humphrey PA. Immunohistochemistry in diagnostic surgical pathology of the prostate. Semin Diagn Pathol 2005;22:88-104.

33 Shah RB, Zhou M, LeBlanc M, et al. Comparison of the basal cell-specific markers, 34betaE1 2 and p63, in the diagnosis of prostate cancer. Am J Surg Pathol 2002;26:1161-8.

34 Ananthanarayanan V, Deaton RJ, Yang XJ, et al. Alpha-methylacyl-CoA racemase (AMACR) expression in normal prostatic glands and high-grade prostatic intraepithelial neoplasia (HGPIN): association with diagnosis of prostate cancer. Prostate 2005;63:341-6.

35 Bostwick DG, Kindrachuck RW, Rouse RV. Prostatic adenocarcinoma with endometrioid features: clinical, pathologic, and ultrastructural findings. Am J Surg Pathol 1985;9:595-609.

36 Epstein JI, Woodruff JM. Adenocarcinoma of the prostate with endometrioid features. A light microscopic and immunohistochemical study of ten cases. Cancer 1986;57:111-19.

37 Green LF, Farrow GM, Ravits JM. Prostatic adenocarcinoma of ductal origin. J Urol 1979:121:303-5.

38 Melicow MM, Pachter MR. Endometrial carcinoma of the prostatic utricle (uterus masculinus). Cancer 1976;20:1715-21.

39 Brinker DA, Potter SR, Epstein Jl. Ductal adenocarcinoma of the prostate diagnosed on needle biopsy: correlation with clinical and radical prostatectomy findings and progression. Am J Surg Pathol 1999;23:1471-9.

40 Humphrey PA. Variants of prostatic carcinoma in prostate pathology. Chicago, IL: ASCP Press, 2003.

41 Melicow MM, Tannenbaum M. Endometrial carcinoma of uterus masculinus (prostatic utricle): report of 6 cases. J Urol 1971;106:892-902.

42 Zaloudek C, Williams JW, Kempson RL. "Endometrial" adenocarcinoma of the prostate: a distinctive tumor of probable prostatic ductal origin. Cancer 1976;37:2255-62.

43 Bostwick DG, Kindrachuck RW, Rouse RV. Prostatic adenocarcinoma with endometrioid features: clinical, pathologic, and ultrastructural findings. Am J Surg Pathol 1985;9:595-609.

44 Ro JY, Ayala AG, Wishnow KI, et al. Prostatic duct adenocarcinoma with endometrioid features: immunohistochemical and electron microscopic study. Semin Diagn Pathol 1988;5:301-1 1

45 Yang XJ, Cheng L, Helpap B, et al. Ductal carcinoma. In: Eble JN, Sauter G, Epstein JI, Sesterhenn IA, eds. Pathology and genetics of tumours of the urinary system and male genital organs. Geneva: WHO (IARC), 2004:199-201.

46 Bock BJ, Bostwick G. Does prostatic ductal adenocarcinoma exist? Am J Surg Pathol 1999;23:781-89. 
47 Zini L, Villers A, Leroy X, et al. Cystic prostate cancer: a clinical entity of ductal carcinomas. Prog Urol 2004;14:411-13.

48 Hameed O, Humphrey PA. Stratified epithelium in prostatic adenocarcinoma: a mimic of high-grade prostatic intraepithelial neoplasia. Mod Pathol 2006; 19:899-906.

49 Perez N, Castillo M, Santos Y, et al. Carcinosarcoma of the prostate: two cases with distinctive morphologic and immunohistochemical findings. Virchows Arch 2005;446:511-16.

50 Wernert $\mathrm{N}$, Lüchtrath $\mathrm{H}$, Seeliger $\mathrm{H}$, et al. Papillary carcinoma of the prostate, location, morphology, and immunohistochemistry: the histogenesis and entity of so-called endometrioid carcinoma. Prostate 1987;10:123-31.

51 Levi AW, Epstein J. Pseudohyperplastic prostatic adenocarcinoma on needle biopsy and simple prostatectomy. Am J Surg Pathol 2000;24:1039-46.

52 Mai KT, Landry DC, Collins JP. Secondary colonic adenocarcinoma of the prostate histologically mimicking prostatic ductal adenocarcinoma. Tumori 2002;88:341-4.

53 Werling RW, Yaziji H, Bacchi CE, et al. CDX2, a highly sensitive and specific marker of adenocarcinomas of intestinal origin: an immunohistochemical survey of 476 primary and metastatic carcinomas. Am J Surg Pathol 2003;27:303-10.

54 Gacucas RJ, Brown RW, Wheeler TM. Verumontanum mucosal gland hyperplasia. Am J Surg Pathol 1995;19:30-6.

55 Samaratunga $\mathbf{H}$, Singh $M$. Distribution pattern of basal cells detected by cytokeratin 34 Beta E12 in primary prostatic duct adenocarcinoma. Am J Surg Pathol 1997;21:435-40.

56 Rioux-Leclercq N, Leray E. Patard J, et al. The utility of Ki-67 expression in the differential diagnosis of prostatic intraepithelial neoplasia and ductal adenocarcinoma. Hum Pathol 2005;36:531-7.

57 McNeal JE, Bostwick DG. Intraductal dysplasia: a premalignant lesion of the prostate. Hum Pathol 1986;17:64-71.

58 Van der Kwast TH, Labrie F, Tetu B. Persistence of high-grade prostatic intraepithelial neoplasia under combined androgen blockade therapy. Hum Pathol 1999:30:1503-7.

59 Montironi R, Mazzucchelli R, Algaba F, et al. Morphological identification of the patterns of prostatic intraepithelial neoplasia and their importance. J Clin Pathol 2000;53:655-65
60 Cohen RJ, McNeal JE, Baillie T. Patterns of differentiation and proliferation in intraductal carcinoma of the prostate: significance for cancer progression. Prostate 2000;43:11-19.

61 Wilcox G, Soh S, Chakraborty S, et al. Patterns of high grade prostatic intraepithelial neoplasia associated with clinically aggressive prostate cancer. Hum Pathol 1998;29:1119-23.

62 Rubin MA, de La Taille A, Bagiella E, et al. Cribriform carcinoma of the prostate and cribriform prostatic intraepithelial neoplasia: incidence and clinical implications. Am J Surg Pathol 1998;22:840-8.

63 Leav I, McNeal JE, Kwan PW-L, et al. Androgen receptor expression in prostate dysplasia (prostatic intraepithelial neoplasia) in the human prostate. An immunohistochemical and in situ hybridization study. Prostate 1996;29:137-45.

64 Dawkins HJS, Sellner LN, Turbett GR, et al. Distinction between intraductal carcinoma of the prostate (IDC-P), high grade dysplasia (PIN), and invasive prostatic adenocarcinoma, using molecular markers of cancer progression. Prostate 2000;44:265-70.

65 Egevad L, Allsbrook WC, Epstein Jl. Current practice of diagnosis and reporting of prostatic intraepithelial neoplasia and glandular atypia among genitourinary pathologists. Mod Pathol 2006;19:180-5.

66 Guo CC, Epstein JI. Intraductal carcinoma of the prostate on needle biopsy: histologic features and clinical significance. Mod Pathol 2006;19:1528-35.

67 Tomlins SA, Rhodes DR, Perner R, et al. Recurrent fusion of TMPRSS2 and ETS transcription factor genes in prostate cancer. Science 2005;310:644-8.

68 Cerveira N, Ribeiro FR, Peixoto A, et al. TMPRSS2-ERG gene fusion causing ERG overexpression precedes chromosome copy number changes in prostate carcinomas and paired HGPIN lesions. Neoplasia 2006;8:826-32.

69 Ullmann AS, Ross OA. Hyperplasia, atypism, and carcinoma in situ in prostatic periurethral glands. Am J Clin Pathol 1967;47:497-504.

70 Oliai BR, Kahane H, Epstein J. A clinicopathologic analysis of urothelial carcinomas diagnosed on prostate needle biopsy. Am J Surg Pathol $2001 ; 25: 794-801$.

71 Cheville JC, Dundore PA, Bostwick DG, et al. Transitional cell carcinoma of the prostate: clinicopathologic study of 50 cases. Cancer 1998;82:703-7.

\section{BNF for Children 2006, second annual edition}

In a single resource:

- guidance on drug management of common childhood conditions

- hands-on information on prescribing, monitoring and administering medicines to children

- comprehensive guidance covering neonates to adolescents

For more information please go to bnfc.org 